\title{
The Changes of Power Spectrum of Electrical Signal in Aloe under Several Damage Conditions
}

\author{
Xiaohui Zhang ${ }^{1}$, Hanqing Sun ${ }^{2}$, Hongbiao $\mathrm{Ma}^{3}$ and Gang $\mathrm{Xi}^{4}$ \\ ${ }^{1}$ College of Electrical Engineering, Henan University of Technology, Zhengzhou \\ 450001, China \\ ${ }^{2}$ Department of Electronic and Information Engineering, Henan University of Animal \\ Husbandry and Economy, Zhengzhou 450044, China \\ ${ }^{3}$ School of Information Engineer, Henan Institute of Science and Technology, \\ Xinxiang 453003, China \\ ${ }^{4}$ Department of Applied Physics, Xi'an University of Technology, Xi'an, \\ 710048, China \\ *Correspondence author: xiaohui.xh@163.com
}

\begin{abstract}
In this paper, the changes of electrical signal in Aloe vera L. under three damage conditions of smoking, burning and broken branches were analyzed through the wavelet soft-threshold de-noising and power spectrum method. The results showed that these methods were feasible, the electrical signal in Aloe vera L. was the low-frequency signal less than $1 \mathrm{~Hz}$ and its amplitude was the $\mu V$ level. The study also found that the changes of amplitude and power spectrum distribution of the electrical signal in Aloe vera L. under smoking condition were small, while the amplitude of the electrical signal was increased clearly and power spectrum distribution of the electrical signal shifted to high-frequency region under burning and broken branches conditions. The power spectrum edge frequency (SEF), spectrum center frequency (SCF) and power spectral entropy (PSE) were defined in order to describe the changes of power spectrum in plant signal. The changes of SEF, SCF, and PSE of power spectrum in Aloe vera L. electrical signal under the conditions of smoke, burning and broken branches were analyzed. It was showed that the SEF, SCF, and PSE of power spectrum could be used to real-time detect responses of plants to environmental stress.
\end{abstract}

Keywords: power spectrum, electrical signal, Aloe vera L., damage

\section{Introductions}

Plant neurobiology is a newly developed discipline in plant physiology, aimed at establishing the structure of information networks within the plant in order to understand how plants respond to environmental stimuli by means of electrochemical signals [1-3]. Electrical, chemical (hormones), hydraulic and mechanical signals constitute the information system of plants in plant neurobiology [4-5]. In information transmission, because the electrical signal can quickly transmit information over long distances within plant[4-7], to transmit information more quickly and effectively compared to chemical signals[4,6,8], and it has proved that electrical signals can affect such central physiological processes as the respiratory metabolism, photosynthesis, stomata conductance, water uptake in physiological processes [4,9-11], therefore, the plant electrical signals become a hot research in recent years. Now it is generally believed that potential fluctuation in the plant is a basic physiological property of plant, plant transmits information flow through this nature to make a specific response to environmental stress $[6,8,12-14]$.

In plant neurobiology research, electrical signal transmission characteristics of the plant have been many studies. Malone et al. [15] (1994) and Stankovic et al. [16] (1998) reported that the signal transmission rate was $7-10 \mathrm{~cm} / \mathrm{s}$ under thermal shock damage. Fromm and 
Bauer [17] (1994) published that signal transmission rate was 3-7 cm / s under cold shock damage. Measurements by Stankovic et al.[16] (1998) was that the EP signal transmission rate in the $40-50 \mathrm{~cm} / \mathrm{min}$ range caused by direct burning of leaf damage. EP signal transmission rate is $20.9 \mathrm{~cm} / \mathrm{s}$ under the broken branches [7]. There are many reports on the signal propagation path [4, 14, 18-19].

Aloe vera $L$. , as fleshy perennial evergreen herb plant, has a wide role in the medical, health, air pollution control and other aspects. Because the electrical signal in Aloe vera L. is collected easily, it has been studied in analyzing plant signal. Volkov et al [20] (2007) studied transmission characteristics of signal within Aloe vera L. induced by local heat stress in detail, electrical signals propagated along all leaves of Aloe vera $L$. plants and possible pathways for electrical signal propagation were studied. However, the study of the variation on the power spectrum of plant electrical signal under the damage conditions has not yet seen. In this paper, the basic characteristic of electrical signal in Aloe vera $L$. was analyzed by wavelet soft-threshold de-noising and power spectrum. Then, the amplitude and power spectrum variation of the electrical signal in Aloe vera $L$. was also studied, which lays the foundation for further study of plant electrical signal.

\section{Material and Method}

\subsection{Material and Signal Collection}

Aloe vera $L$. was brought from the flower market, which the height is $45 \mathrm{~cm}$ and 8 leaves. Because the electrical signal in plant is very small, the collecting system about plant electrical signal must have some characteristics such as high input impedance $\left(>10^{10} \Omega\right)$, high common mode rejection ratio $(>120 \mathrm{~dB})$, low noise $(<1 \mu \mathrm{V})$ and low drift etc. According to these characteristics, BL-420S biological function experiment system (designed by Chengdu TME Technology Co, Ltd.) was used. This system can amplify and filter the biological signal, process the signal through analog digital conversion to digitization, transmit the digitization signal to computer.

In order to obtain more accurate plant electrical signal, the patch electrode was used in this paper, the electrode is AMBU ECG electrode of silver. Its diameter is $3.4 \mathrm{~cm}$. The structure is made up of lining materials, conductive paste and electrode buckle, etc. It has high sensitivity, low noise and its surface doesn't need to pretreatment. In Figure 1, A is positive electrode and $\mathrm{B}$ is negative electrode, $\mathrm{C}$ is reference electrode.The positive and negative electrodes are pasted on both sides of plant leaf, the distance between two electrodes is $30 \mathrm{~cm}$, reference electrode connects ground. Stable plant signal can be obtained accurately.

In the experiment, Aloe vera $L$. was put into Faraday cage $(60 \mathrm{~cm} \times 60 \mathrm{~cm} \times 60 \mathrm{~cm})$. The electrical signal in Aloe vera $L$. under normal growth condition was collected as control (CK), and then Aloe vera $L$. suffered smoke, burning and broken branches processing, the electrical signal in Aloe vera $L$. was collected (The distance is $15 \mathrm{~cm}$ ) at $25^{\circ} \mathrm{C}$, the average humidity was $45 \%-50 \%$ and light intensity was 5001x. While collecting the signal, the second channel of the signal measurement system is the inputting channel of plant signal and the third channel is hung in the air (as the test channel of noise to confirm the plant signal). The characteristic of the signal was analyzed by computer. The sampling frequency is $2 \mathrm{~K} \mathrm{~Hz}$, the low-pass filter's frequency is $500 \mathrm{~Hz}$, and high-pass filter's frequency is $0.053 \mathrm{~Hz}$. The connecting method is showed as Figure 1. 


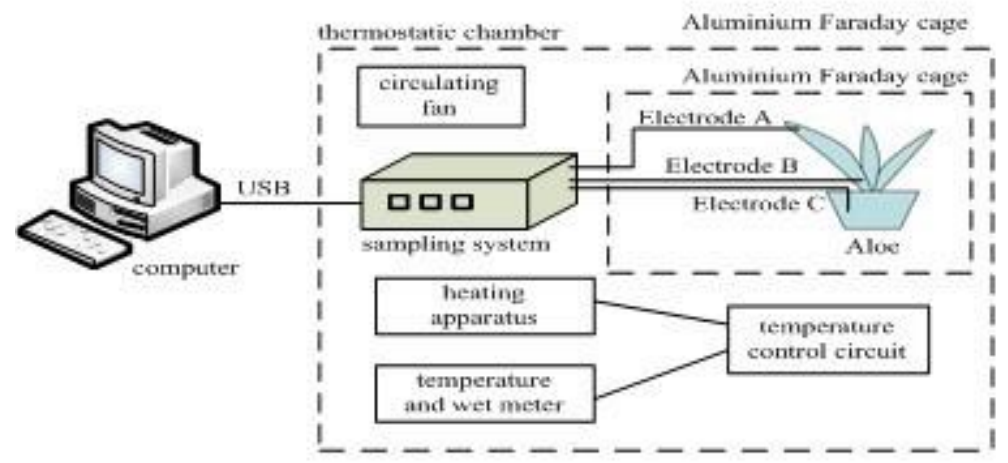

Figure 1. Schematic Diagram of Experiment Device

\subsection{Data progressing}

\subsubsection{Wavelet de-noising}

In the experimental process, although using grounding, shielding and other measures to minimize interference from outside, the signal collected still contained some noise. In order to obtain as accurately as possible the spectral characteristics of Aloe vera L. signal, electrical signals collected were de-noised by wavelet transform in this paper ${ }^{[21]}$.

Wavelet transform (WT) is defined as:

$$
W T_{f}(a, b)=\frac{1}{\sqrt{a}} \int_{-\infty}^{+\infty} f(t) \psi^{*}\left(\frac{t-b}{a}\right) d t
$$

Where, $\psi$ is based-wavelet. $f(t)$ is the signal which contains the noise, $a$ is scaling factor, $b$ is displacement factor, and $a, b \in R, a \neq 0$. The signals are decomposed into two parts including approximation coefficient and detail coefficient by wavelet transforming. De-noising is a threshold quantization processing on the detail coefficients of each decomposition scale to select a threshold value, and then the signal is reconstructed.

Than the wavelet coefficients are processed by soft threshold. When selecting threshold, the noise standard deviation $\sigma$ of original signal is determined with estimation method. It is showed in equation (2):

$$
\sigma=\frac{\text { median }\left(\left|d_{i}(k)\right|\right)}{0.6745}
$$

Where, $i$ is wavelet decomposition scale, median is the order of the value of MATLAB. Unified threshold of Donoho and Johnstone is adopted in this paper [22].

$$
t h r=\sigma \sqrt{2 \log (n)}
$$

According to the equation (3) the threshold of each scale is calculated, then wavelet detail coefficients are quantized by using soft-threshold. When the absolute value of wavelet coefficients is less than the threshold, the wavelet coefficient is zero. Otherwise, the wavelet coefficient is the value of subtracting the threshold from them. It is showed in equation (4):

$$
d_{i, t h r}=\left\{\begin{array}{l}
0,\left|d_{i}\right|<t h r \\
{\left[\operatorname{sgn}\left(d_{i}\right)\right]\left(\left|d_{i}\right|-t h \eta\right),\left|d_{i}\right| \geq t h r}
\end{array}\right.
$$

Where, $d_{i, t h r}$ is the wavelet coefficient after threshold.

The signal $f(t)$ is got from inverse wavelet transform. The equation is as follows: 


$$
f(t)=\frac{1}{C_{\psi}} \int_{-\infty}^{+\infty} \int_{-\infty}^{+\infty}\left[\left(W_{\psi} f\right)(a, b)\right] \psi_{a, b}(x) \frac{d a}{a^{2}} \mathrm{~d} b
$$

\subsubsection{Power Spectral Analysis}

Typically, the spectrums of useful signal and noise are different, so power spectrum estimation is an effective way to extract useful signal on the noise background. Spectrum estimation methods are composed of classical spectrum estimation (non-parameter estimation) and modern spectral estimation (parameter estimate).The former includes BT, Welch, Bartlett and periodogram method, the latter includes Levinsio and Burg method. Classical spectrum estimation is not only simple for calculation of short-term signal, but also periodogram method has the higher resolution and reliability on the basis of long data series. Therefore periodgram method is used to analyze plant electrical signals in this paper [23].

The power spectrum of stationary random process is estimated by periodogram defined as follows:

$$
S_{P}\left(\mathrm{e}^{\mathrm{j} \omega}\right)=\frac{1}{N}\left|\sum_{n=0}^{N-1} x(n) \mathrm{e}^{-\mathrm{j} \omega n}\right|^{2}
$$

The first, a window function is added to data.The rectangular window is used in this paper as follows:

$$
w(n)=R_{N}(n)=\left\{\begin{array}{l}
1,0 \leq n \leq N-1 \\
0, \text { other }
\end{array}\right.
$$

The power spectrum can be obtained as:

$$
S_{\mathrm{M}}\left(\mathrm{e}^{\mathrm{j} \omega}\right)=\frac{1}{N}\left|\sum_{n=0}^{N-1} x(n) w(n) \mathrm{e}^{-\mathrm{j} \omega n}\right|^{2}
$$

The parameters of power spectrum analysis in this paper are as follows:

(1) Spectral edge frequency (SEF). The power of the signal between $\mathrm{OHz}-\mathrm{SEF}$ accounts for $95 \%$ of the total power.

(2) Spectral center frequency (SCF). The formula is

$$
f_{g}=\frac{\sum_{f=f_{1}}^{f_{2}}[P(f) \times f]}{\sum_{f=f_{1}}^{f_{2}} P(f)}
$$

Where: $f_{g}$ is SCF, $f_{1}$ to $f_{2}$ for the frequency range, $P(f)$ for the signal power, $f$ is frequency.

(3) Power spectral entropy (PSE). PSE is defined as

$$
H_{f}=-\sum P_{f} \ln P_{f}
$$

Where, $f$ is the frequency, $P_{f}$ is the percentage of power at the frequency $f$ in the entire spectrum of power.

\section{Results and Discussion}

\subsection{The Time-Domain Features of Electrical Signals in Aloe Vera L.}

Figure 2 (a), (b), (c) and (d) are the time-domain waveforms of the electrical signal in Aloe Vera $L$. under normal growth (CK), smoking, burning and broken branches respectively. These Figures show that the electrical signal in Aloe Vera L. is a kind of random signal. In order to better analysis the basic characteristics of these electrical signals, wavelet coefficients are calculated by $\mathrm{db} 7$ wavelet at 7 scale decomposition. The transform result is showed in 
Figure 3. It can be seen from Figure 3 that the high-frequency detail is broken down in the high-frequency scale (detail coefficients), which reflects noise component of the measured signals, and the mutation point of the electrical signal also is broken out which corresponds to action potential.

The electrical signal in Aloe vera $L$. is de-noised through wavelet soft-threshold method, reconstructed through inverse wavelet transform. The result is showed in Figure 4(a), (b), (c) and (d). It can be seen from the Figure 4 that the original features of the signal is better retained, the high frequency noise is filtered out. De-noised signal has better smoothness than the original signal. Hence, wavelet analysis has a better de-noising effect for the electrical signal in Aloe vera $L$.
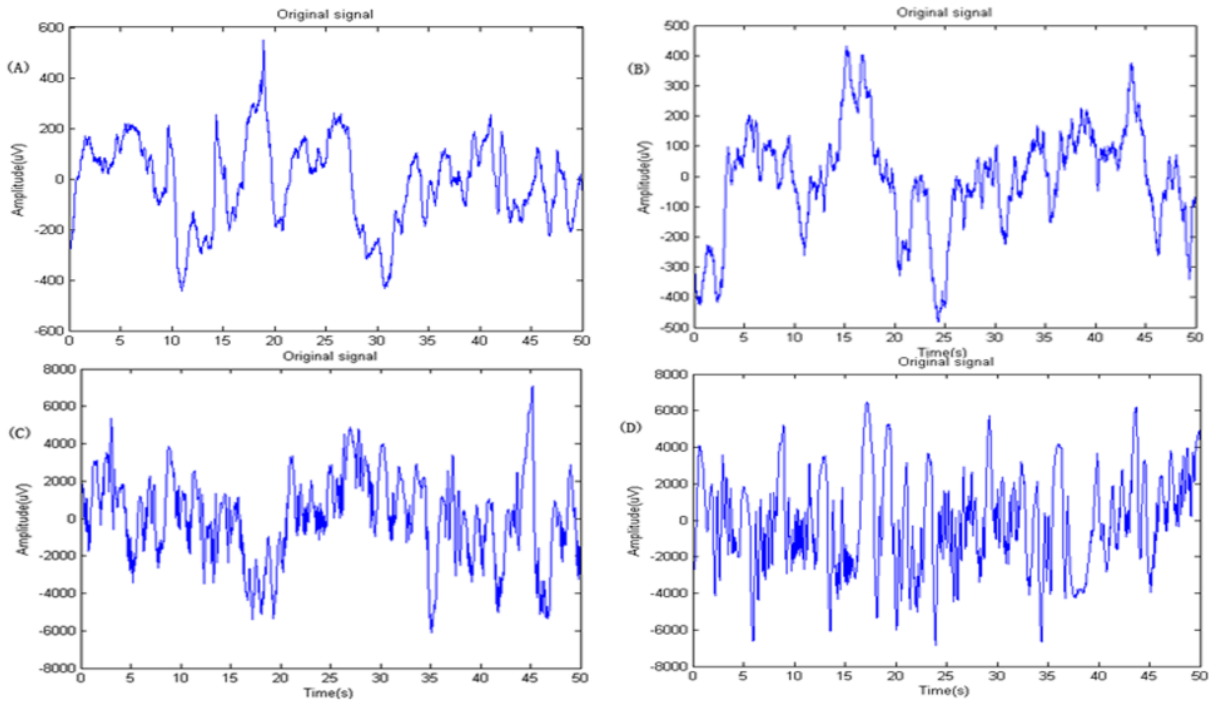

Figure 2. Electrical Signal in Aloe Vera L. Under Different Damage Conditions (A)CK

(B) Smoking

(C) Burning

(D) Broken branches

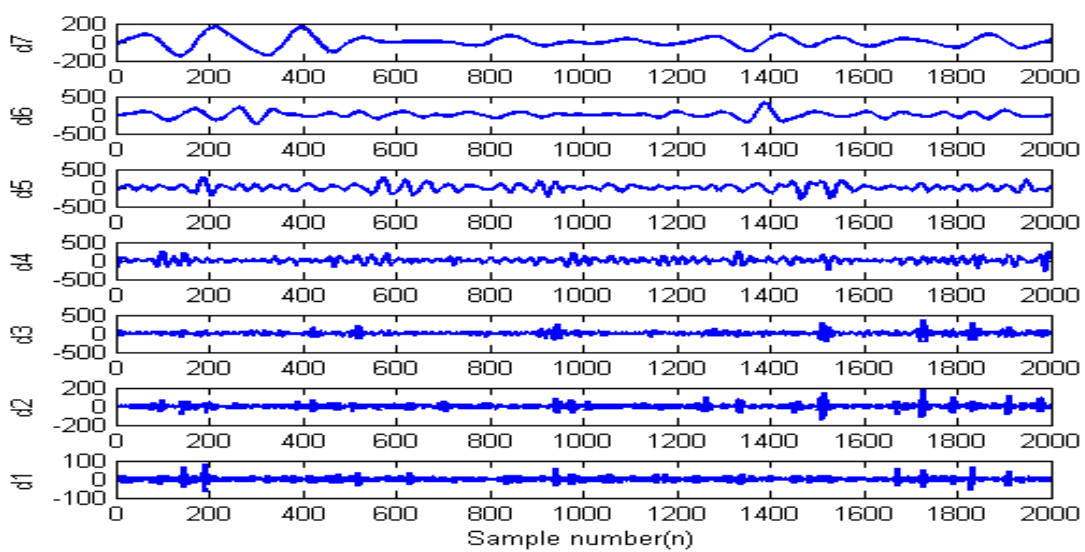

Figure 3. The Diagram of 7 Scale Decomposition (D1 D7 is the Detail Coefficients) 

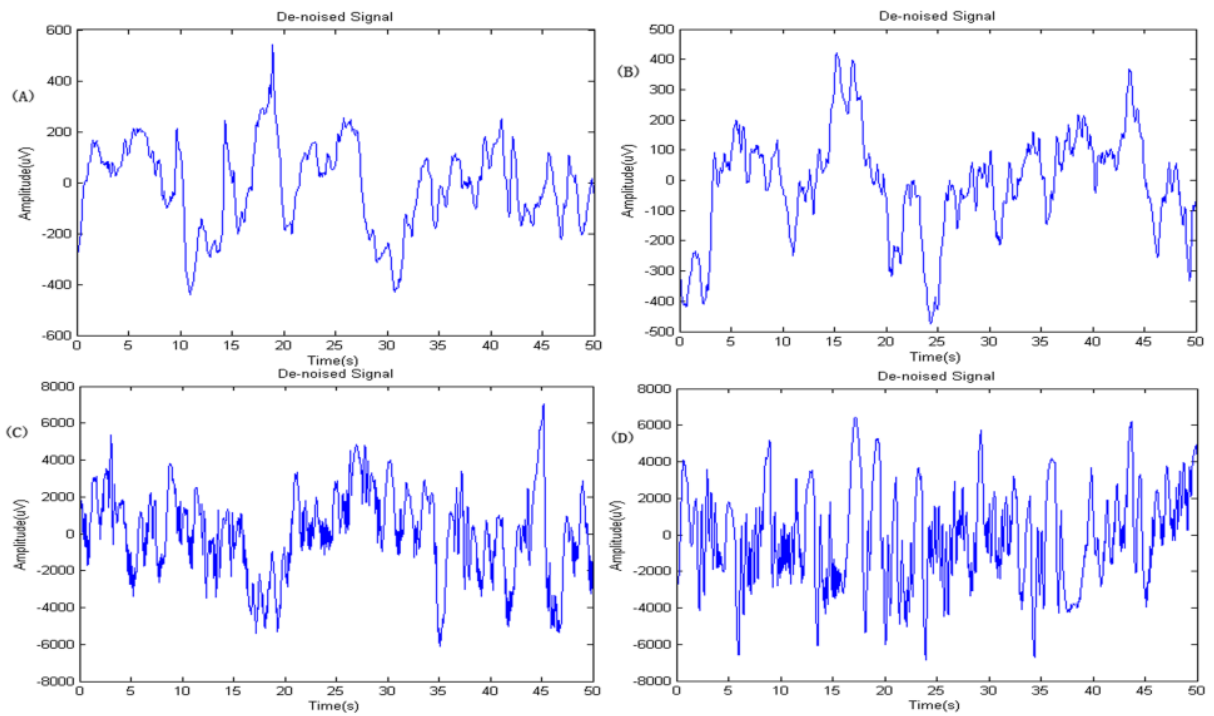

Figure 4. The De-Noised Electrical Signal in Aloe Vera L. Under Different Damage Conditions
(A)CK
(B) Smoking
(C) Burning
(D) Broken branches

\subsection{The Amplitude of Electrical Signal in Aloe Vera L. Under Damage Conditions}

The amplitudes of electrical signal in Aloe vera $L$. under damage condition form Figure 4 are showed in Figure 5. It can be seen that the electrical signal in Aloe vera $L$. is in the $\mu \mathrm{V}$ level under normal condition. The change of signal amplitude is small when smoking, while the maximum of the amplitude is about $7000 \mu \mathrm{V}$ when burning and broken branches, which is larger than under normal and smoking condition. This indicates that the amplitude of electrical signal on near leaf is greatly increased by the stimulation of burning and broken branches.

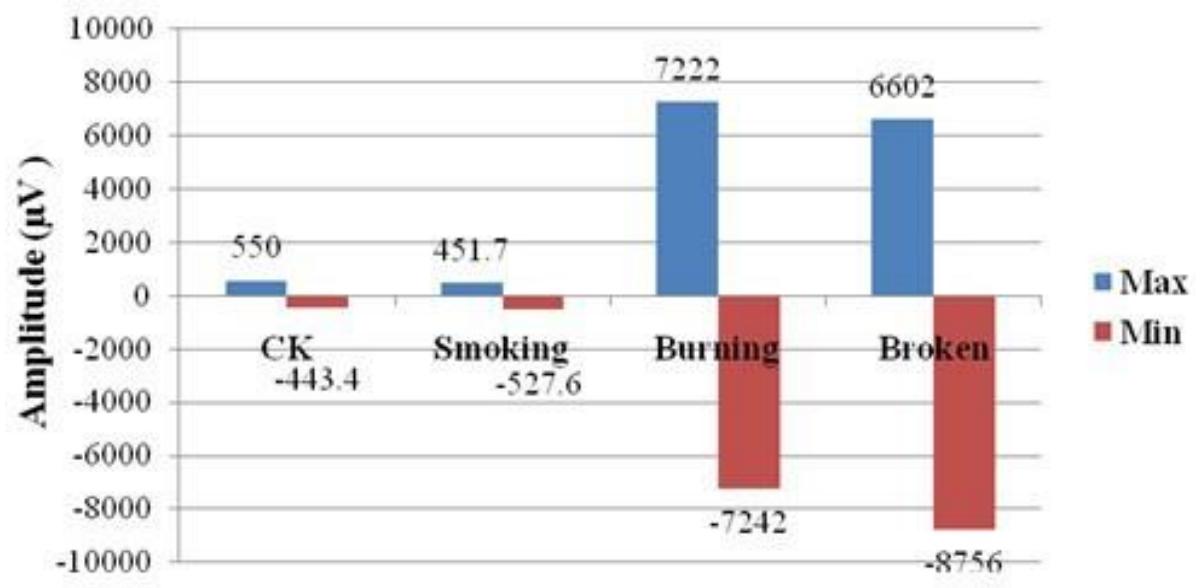

Figure 5. The Amplitude of Electrical Signal in Aloe Vera L. Under Different Damage

\subsection{The Power Spectrum of Signal under Different Damage}

Power spectrum of de-noised signals is analyzed by periodogram method using Matlab R2008 software. The results are shown in Figure 6. 

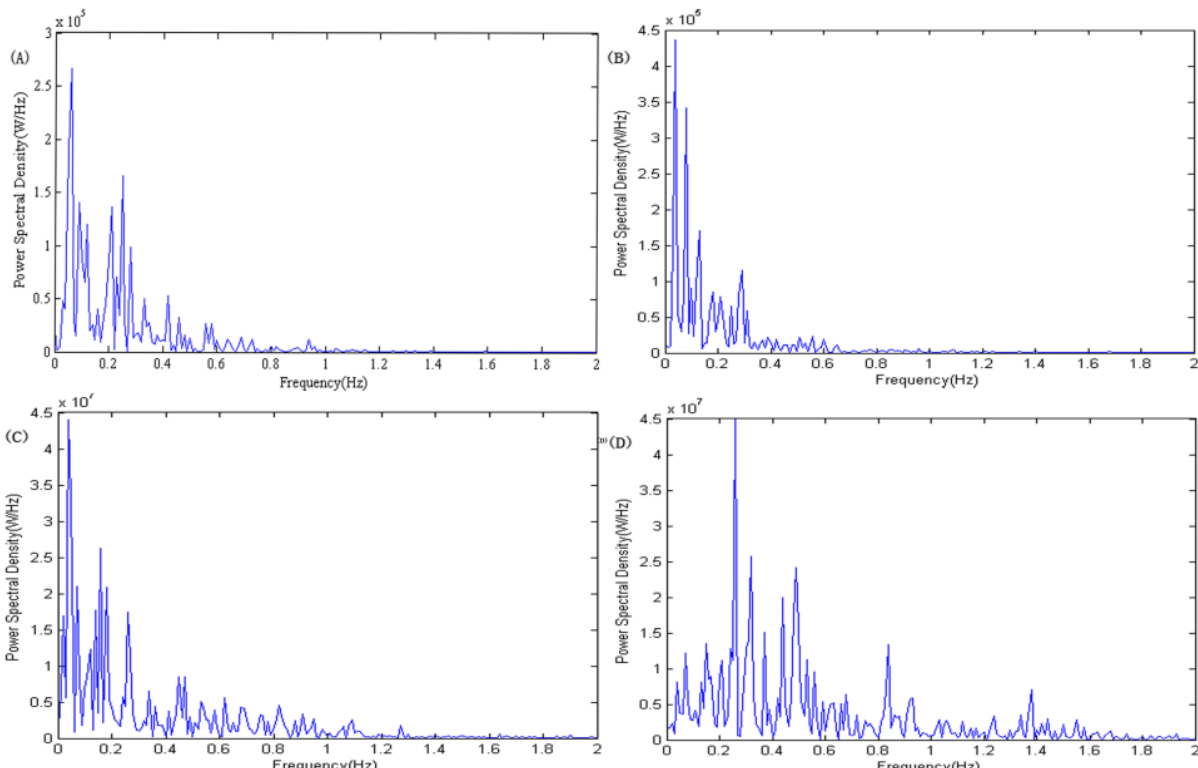

Figure 6. The Power Spectrum of Signal under Different Damage Conditions
(A)CK
(B) Smoking
(C) Burning
(D) Broken branches

It can be seen form Figure 6that the frequency of electrical signal in Aloe vera $L$. is in $0 \sim 1 \mathrm{~Hz}$ and the power spectrum of electrical signal changes under different damage conditions. When normal growth and smoking, the change of signal energy is small, when burning and broken branches, the change of signal energy is great, and the distribution of power spectrum of electrical signal is also different.

\subsubsection{The Changes of SEF and SCF}

In order to illustrate the difference of signal power spectrum under damage conditions, the SEF and SCF of power spectrum of Aloe vera $L$. signals under various conditions are calculated, the results are shown in Figure 7. It is showed in Figure 7 that the SEF of signal in control is $0.8 \mathrm{~Hz}$, indicates that the power distribution of Aloe vera $L$. is in the range of $0-1 \mathrm{~Hz}$. When smoking, burning and broken branches, the SEF is $0.8,1.2$ and $1.7 \mathrm{~Hz}$ respectively. These results show that there is no significant change of signal power spectrum when smoking, but signal power spectrum when burning and broken branches extends to the high frequency, the extension of power spectrum when broken branches is larger.

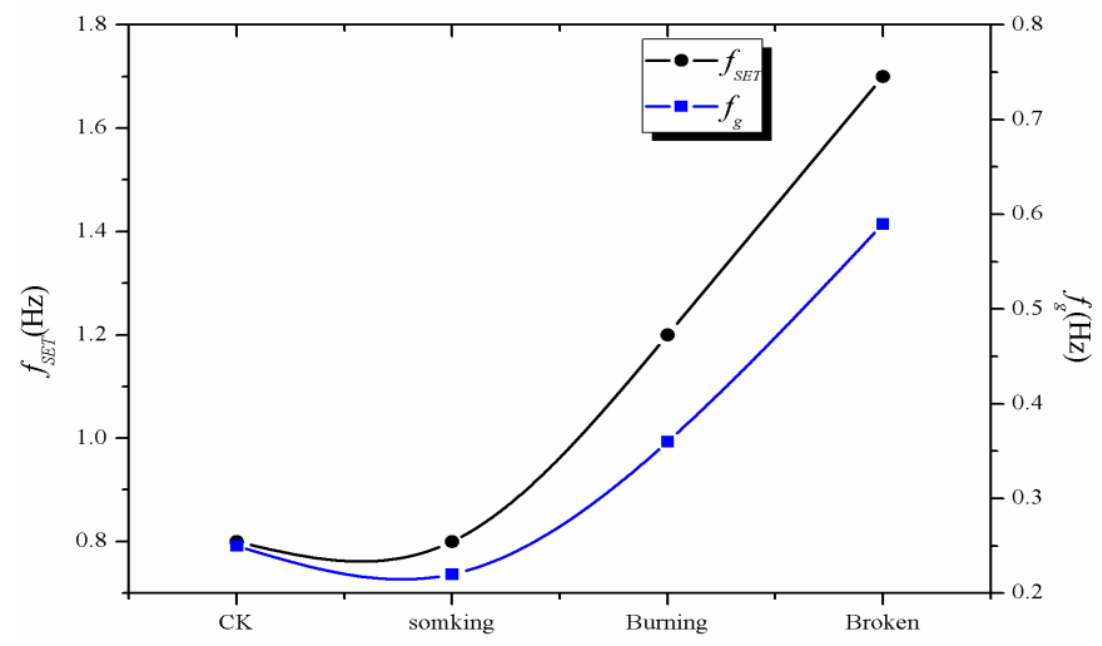

Figure 7. The Changes of SEF and SCF of the Signal under Different Damage Conditions 


\subsubsection{The Changes of PSE}

The changes of PSE of power spectrum in Aloe vera L. signals under different damage conditions are shown in Figure 8. When burning and broken branches the PSE of signal increases significantly compared with the control, the rates of increase reach $19 \%$ and $42 \%$ respectively.

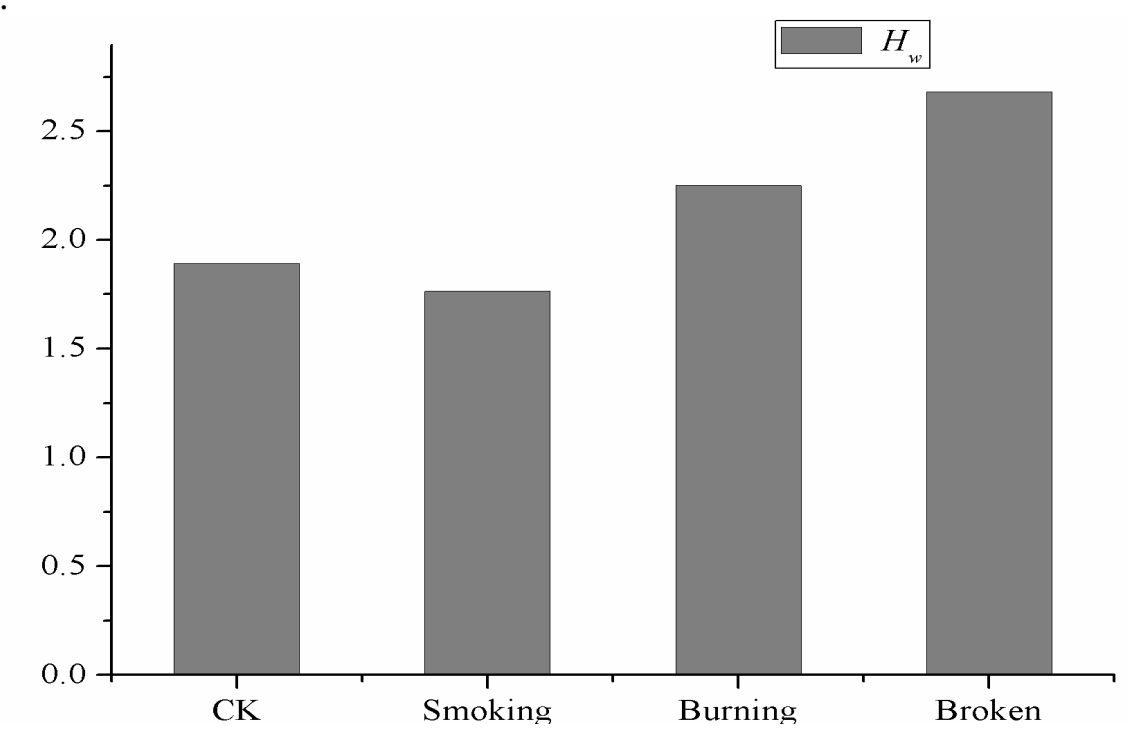

Figure 8. The Changes of PSE under Different Damage Conditions

\section{Discussion}

Electrical signals in plants include the local potential (LP), action potential (AP) and the variation of potential (VP). LP is the non-conductive potential changes in plant when stimulation. External stimulation will be converted to membrane potential in plant through the LP. When the membrane potential exceeds a certain threshold would lead to AP and VP. AP and VP spread in the form of electrical impulses in plant tissues and organs. The electrical signal of Aloe vera $L$. showed in Figure 4 includes VP and AP. To the generation of electrical signals on the plant, transmission, reception and transformation mechanism, many researchers agree with the idea that a certain stimuli receptor must be present at the cell membrane, and that a transient polarization, induced by specific ion fluxes through this membrane, is the ultimate agent of the electrical potential signal generation [1, 2, 13, 24]. An action potential is an electrical signal that spreads quickly among plant tissues and organs, traveling at a relative high speed and constant amplitude $[4,9,14,18,20]$, its duration is of the order of milliseconds and are generated by a stimulus that requires a specific threshold for its initiation $[7,25]$. In this paper, when the smoking, there is little change of the signal amplitude and the distribution of power spectrum compared with control, indicates that the signal is mainly LP. When the burning and broken branches, the signal amplitude has changed dramatically, this change is due to the generation and dissemination of AP and VP. The cells received over the AP signal to regulate the changes of $\mathrm{Ca}^{2+}$ concentration in cytoplasm through the ion channel opening and closing in cell membrane enable an ion flux between the cytosol and the extracellular microenvironment, which creates electrical potential differentials across the membrane $[26,31]$. Variations in $\mathrm{Ca}^{2+}$ concentrations in the cytosol modify the catalytic activity of the enzyme calmonduline [27-28] as well as the activity of different protein kinases (CDPKs) dependent on $\mathrm{Ca}^{2+}[29-31]$, physiological changes are induced. Thus, the sharp increase of the signal amplitude received by cell would have dramatic impact on ion channel regulation in the cell membrane and physiological changes.

In addition to amplitude changes, the signal power spectrum distribution of Aloe vera $L$. have also changed when burning and broken branches. To quantitative describe the changes in plant signal power spectrum, the concept of SEF and SCF was put forward in this paper. 
The smaller the SEF, the more close to the low frequency in power spectrum distribution, and the deeper cells are inhibited. On the contrary, shows the cells more "exciting". SCF not only can reflect the distribution of signal power spectrum, can also reflect the migration of the power spectrum under different conditions, therefore, SEF or SCF can be used as cell "excitement" of the measure. This result shows that when smoking, the SEF and SCF of signals have little change, the great changes of the SEF and SCF have taken place when burning and broken branches, the biggest change occurred when the broken branches. It is indicated that the cells in different parts of plants will respond to irreversible damage, and the greater the damage, cells respond more strongly. Because the signal power spectrum contains various frequencies of AP and VP signals, an action potential is caused by the movement of ions across the plasma membrane, resulting from a change in its specific permeability to different ions, with transient variations of the cytosol and its external environmental and electrochemical potential $[19,24,32]$, a variation potential consists of a transient change in membrane potential (depolarization and subsequent slow repolarization), therefore, the signal power spectrum distribution is actually produced by complex transmembrane transport of ions and membrane potential changes, it reflects complexity of ion movement and the changes of membrane potential. The results in this paper show that the power spectrum of the electrical signal in Aloe vera $L$. expands to the high-frequency region when irreversible injury, it indicates that the frequency components of the signal increase and the transmembrane transport of ions and membrane potential distribution are more complex. In order to quantitative describe the complexity of power spectrum distribution of plant electrical signal and the changes of power spectrum in the distribution, power spectral entropy (PSE) of the signals was defined from Shannon entropy, as a measure of complexity of power spectrum distribution. The narrower of power spectrum, the smaller the PSE, it indicates that there is obvious oscillation rhythm in signals, the complexity of signal is small. Conversely, the more flat the power spectrum, PSE greater, indicates that the signal is more complex. Figure 8 shows that the PSE of electrical signal in Aloe vera L. significantly increases compared with the control when burning and broken branches, the rate of increase is greatest, illustrates the complexity of signal power spectrum is maximum. It can be seen that the changes of PSE, SEF and SCF are synchronized compared SEF, SCF with PSE. The consistency of the change of PSE, SEF and SCF indicates that the changes of SEF, SCF and PSE are adaptive responses of plants to injury. Because the changes in plant electrical signal are earlier than the various physiological changes and the morphological changes [2, 33-34]. Thus, responses of plant to stress can quickly and accurately judge according to signal changes. In this paper, the changes of the electrical signal power spectrum can be quantitative described from SEF, SCF and PSE, these parameters could be used as early indicators of real-time assessment of plant responses to stress.

\section{Conclusion}

Wavelet soft threshold de-noising method can effectively reduce the noise signal and better retain the characteristics of the original electrical signal in Aloe vera $L$. The frequency range of electrical signal in Aloe vera $L$. is in $0 \sim 1 \mathrm{~Hz}$. The change of amplitude and power spectrum of electrical signal in Aloe vera $L$. is small when smoking. The change of amplitude of electrical signal in Aloe vera $L$. is obviously when burning and broken branches, the characteristic parameters of the power spectrum SEF and SCF shift to high-frequency region, PSE increases. SEF, SCF and PSE of plant electrical signal can be used as an early indicator of real-time assessment of plant response to environment stress. 


\section{Acknowledgments}

This study was funded by the national natural science foundation of china (31471412) and the Science and Technology Research Key Project of Henan Education Department (14B416001) and Henan University of technology high-level talent funded project (2013BS063).

\section{References}

[1] F Baluska, S Mancuso, D Volkmann and P Barlow, "Root apices as plant command centers: the unique 'brain-like' status of the root apex transition zone", Biol Brat. (2004), pp. 59:1-13.

[2] E. D. Brenner, R. Stahlberg and S. Mancuso, "Plant neurobiology : an integrated view of plant signaling", Trends Plant Sci, vol. 11, no. 8, (2006), pp. 413-421.

[3] A Trewavas, "Green plants as intelligent organisms", Trends Plant Sci, vol. 10, no.9, (2005), pp. 414-9.

[4] J. Fromm and S. Lautner, "Electrical signals and their physiological significance in plants",

Plant Cell Environ, vol. 30, no. 3, (2007), pp. 249-57.

[5] P. Gil, L. Gurovich, B. Schaffer, N. García, S. Rey and R. Iturriaga, "Electrical signaling, stomatal conductance, ABA and ethylene content in avocado trees in response to root hypoxia", Plant Signal Behav, vol. 4, (2009), pp. 100-8.

[6] P. Gil, L. Gurovich, B. Schaffer, J. Alcayaga, S. Rey and R. Iturriaga, "Root to leaf electrical signaling in avocado in response to light and soil water content", J Plant Physiol, vol. 165 no. 10, (2008), pp. 1070-8.

[7] P. Oyarce and L. Gurovich, "Evidence for the transmission of information through electric potentials in injured avocado trees", J Plant Physiol, (2010), Available online 13.

[8] L. Gurovich and P. Hermosilla, "Electric signaling in fruit trees in response to water applications and light-darkness conditions", J Plant Physiol, vol. 166, no. 3, (2009), pp. 290-300.

[9] K. Trebacz, H. Dziubinskav and E. Krol, "Electrical signals in long-distance communication in plants", In: Baluska F, Mancuso S, Volkmann D, editors. Communication in plants - neuronal aspects of plant life[M]. Berlin, Heidelberg: Springer-Verlag; (2006), pp. 277-90.

[10] C Koziolek, Grams TEE and U. Schreiber, "Transient knockout of photosynthesis mediated by electrical signals", New Phytol, vol. 161, no. 3, (2003), pp. 715-22.

[11] E. Masi, M. Ciszak, G. Stefano, L. Renna, E. Azzarello, C. Pandolfi, A. Mugnai, F. Baluska,T. Arecchi and S. Mancuso, "Spatiotemporal dynamics of the electrical network activity in the root apex", Proc Nat Acad Sci USA, vol. 106, no. 10, (2009), pp. 4048-53.

[12] J. Fromm and H. Fei, "Electrical signaling and gas exchange in maize plants of drying soil", Plant Sci, vol. 132, no. 2, (1998), pp. 203-13.

[13] A. Volkov, T. Adesina, V. Markin and E. Jovanov, "Kinetics and mechanism of Dionaea mus-cipula trap closing", Plant Physiol, vol. 146, no. 2, (2008), pp. 694-702.

[14] S. Lautner, T. Erhard, R. Matyssek and J. Fromm, "Characteristics of electrical signals inpoplar and responses in photosynthesis", Plant Physiol, vol. 138, no. 4, (2005), pp. 2200-2209.

[15] M. Malone, J. Alarcon and L. Palumbo, "A hydraulic interpretation of rapid, long-distance wound signaling in the tomato", Planta, vol. 193, (1994), pp.181-5.

[16] B. Stankovic, D. Witters, T. Zawadzki and E. Davies, "Action potentials and variation potentials in sunflower: an analysis of their relationships and distinguishing characteristics", Physiol Plant, vol. 103, (1998), pp. $51-8$.

[17] J. Fromm and T. Bauer, “Action potentials in maize sieve tubes change phloem translocation”, J Exp Bot, vol. 45, (1994), pp. 463-9.

[18] E. Davies, "New functions for electrical signals in plants", New Phytol, vol. 161, no. 3, (2004), pp. 607-10.

[19] A. Volkov, "Green plants: electrochemical interfaces", J Electroanal Chem, vol. 483, (2000), pp. 150-6.

[20] A. G. Volkov, R. D. Lang and I. Maia, "Volkova-Gugeshashvili. Electrical signaling in Aloe vera induced by localized thermal stress', Bioelectrochemistry, vol. 71, no. 2, (2007), pp. 192-197.

[21] T. Qi ao, Y. Ni uan and X. Ning, "Research of weak electrical signals based on wavelet de-noising", Life Science Instruments, vol. 7, no. 10, (2009), pp. 20-24.

[22] D. L. Donoho, "De-moise by soft-thresholding", IEEE Trans on IT, vol. 14, no. 3, (1995), pp. 613-627.

[23] W. Xin and P. Zhang, "The analysis on the window function of periodogram power spectrum estimate", Modern electronic technology, vol. 28, no. 3, (2005), pp. 14-15.

[24] A. Volkov and C. Brown, "Electrochemistry of plant life. In: Volkov A, editor. Plant: theory and methods", Berlin, Heidelberg: Springer; (2006), pp. 437-59.

[25] S. Mancuso, "Hydraulic and electrical transmission of wound-induced signals in Vitis vinifera", Aust J Plant Physiol, vol. 26, (1999), pp. 55-61.

[26] V. Demidchik, A. Sokolik and Y. Vladimir, "Electrophysiological characterization of plant cation channels", In: Volkov A, editor. Plant electrophysiology: theory and methods. Berlin/Heidelberg: Springer; (2006), pp. 173-86.

[27] A. Vian, C. Vian, R. Schantz, G. Ledoigt, M. Franchisse Desbiez and J. Julien, "Is membrane potential involved in calmodulin gene expression after external stimulation in plants? FEBS Lett vol. 380, (1996), pp. 93-6. 
[28] J. Leon, E. Rojo and J Sánchez-Serrano, "Wound signaling in plants", J Exp Bot, vol. 52, no. 354, pp. 20011-9.

[29] P. White and M. Broadley, “Calcium in plants', Ann Bot, vol. 92, no.4, (2003), pp. 487-511.

[30] A. Ludwing, T. Romeis and J. Jones, “ CDPK-mediated signaling pathways: specificity and cross-talk”, J Exp Bot, vol. 55, no. 395, (2004), pp. 181-8.

[31] S. Medvedev, "Calcium signaling system in plants", Russ J Plant Physl, vol. 52(no. 2, (2005), pp. 248-70.

[32] A. Gelli and E. Blumwald, "Hyperpolarization-activated $\mathrm{Ca}^{2+}$-permeable channels in the plasma membrane of tomato cells", J Membr Biol, vol. 155, (1997), pp. 35-45.

[33] W. Peter, "Barlow. Reflections on "plant neurobiology", BioSystems, vol. 92, no. 2, (2008), pp. 132-147

[34] X. Yan, Z. Wang, L. Huang, C. Wang, R. Hou, Z. Xu and X. Qiao, "Research progress on electrical signals in higher plants", Progress in Natural Science, vol. 19, no. 5, (2009), pp. 531-541. 
International Journal of Control and Automation Vol. 9, No. 6 (2016) 